\title{
On the Progenitor of SNR 0509-67.5
}

\author{
Ashley Pagnotta and Bradley E. Schaefer \\ Department of Physics \& Astronomy, Louisiana State University \\ 202 Nicholson Hall, Tower Dr., Baton Rouge, LA 70803 \\ email: pagnotta@phys.lsu.edu
}

\begin{abstract}
We have used three independent methods to determine an accurate and precise geometric center of SNR 0509-67.5, at RA=05:09:31.208, DEC $=-67: 31: 17.48$ (J2000). This supernova, which occurred approximately 400 years ago in the Large Magellanic Cloud, was confirmed to be a Type Ia by Rest et al. (2005); Rest et al. (2008) based on spectra of a light echo from the eruption. If this supernova had a single-degenerate progenitor system, we would see the "leftover" companion star within a certain distance of the remnant's center. Accounting for an offset due to enhanced ISM in the west-southwest quadrant of the remnant, we find the eruption position to be at $\mathrm{RA}=05: 09: 30.976, \mathrm{DEC}=-67: 31: 17.90$; the error circle which should contain any possible ex-companion star has a radius of $1.60^{\prime \prime}$ for $99.73 \%$ (3-sigma) containment. This accounts for the proper motion of the stars, the possibility of kicks from the supernova, and asymmetries in the explosion and remnant expansion. We find no possible ex-companion stars within this ellipse, to a limiting magnitude of $\mathrm{V}=26.9$ : there are no red giants, which precludes symbiotic progenitors, no subgiants, which when combined with the lack of red giants precludes recurrent nova progenitors, and no main sequence stars with mass greater than 1.16 solar masses (V brighter than $22.7 \mathrm{mag}$ ), which precludes persistent supersoft X-ray source progenitors. Indeed, all published SD models are eliminated, so we conclude that this particular Type Ia supernova had a double-degenerate progenitor.
\end{abstract}

Keywords. supernovae — supernova remnants: individual (SNR 0509-67.5)

\section{Introduction}

Type Ia supernovae (SNe Ia) are generally accepted to occur when an accreting carbonoxygen white dwarf (WD) reaches the Chandrasekhar mass limit and undergoes a thermonuclear explosion. The standardizable nature of the explosions (Hamuy et al. 1996) makes them a useful tool for measuring cosmological distances (Riess et al. 1998; Perlmutter et al. 1999), but the nature of the progenitor systems remains a mystery. Various candidate systems have been proposed (c.f. Table 1), and these can be divided into singledegenerate (SD) and double-degenerate (DD) sub-classes. In DD systems, both WDs will be destroyed by the SNe Ia explosion, but SD systems will leave behind an identifying ex-companion star after the lone WD explodes. We can look for these ex-companions by searching in the center of known Ia supernova remnants (SNRs).

This method was first used by Ruiz-Lapuente et al. (2004) for Tycho's SN (SN 1572). They identified their Star G as the subgiant ex-companion of the SN based on its location near the center of the remnant and the high radial velocity. González Hernández et al. (2009) observed a high abundance of nickel and cobalt, indicative of elemental enhancement from the explosion. This identification has been challenged, however, by Kerzendorf et al. (2009) whose analysis of the high resolution spectra show nothing unremarkable about Star G's rotation, radial velocity, or proper motion, and note that many of the uncertainties arise from the fact that the distance to Star G is not well known.

We avoid some of these problems by applying this method to the four known SNe Ia in the Large Magellanic Cloud (LMC), which has three main advantages: the stars are all at 


\begin{tabular}{lllll}
\hline \hline Candidate Class & $P_{\text {orb }}($ days $)$ & $V_{\text {orb }}(\mathrm{km} / \mathrm{s})$ & Surviving Companion & $\begin{array}{c}\text { V range in } \\
\text { LMC (mag) }\end{array}$ \\
\hline Double-degenerate & - & - & None & - \\
Recurrent Nova & $0.6-520$ & $70-350$ & Red Giant or Subgiant & $16-22$ \\
Symbiotic Star & $245-5700$ & $70-200$ & Red Giant & $16-19$ \\
Persistent Supersoft Source & $0.14-4.0$ & $200-500$ & Subgiant or $>1.16 M_{\odot}$ MS & $19-22.7$ \\
\hline
\end{tabular}

Table 1. Possible progenitor classes and their characteristics in the LMC. These values are calculated for Roche lobe-filling companions at the accepted LMC distance modulus of $m-M=18.50 \pm 0.10 \mathrm{mag}$. The potential ex-companion stars are all quite bright, and very easy to observe (if they exist) with modern telescopes.

a known distance (we assume $m-M=18.50 \pm 0.10 \mathrm{mag}$ ), the extinction is known to be low (Zaritsky et al. 2004), and the LMC star fields are much less crowded than galactic fields are. In particular, we look at LMC SNR 0509-67.5. This 400-year-old remnant is known to come from a Ia based on X-ray spectral observations (Hughes et al. 1995) as well as spectra of the light echo from the original explosion (Rest et al. 2005; Rest et al. 2008). Because of the location in the LMC, we can accurately describe the appearance of each possible ex-companion star (c.f. Table 1) and search based on those criteria.

\section{Observations}

We used archival Hubble Space Telescope (HST) images to define the center of the remnant and then search near that center for possible ex-companion stars. $\mathrm{H} \alpha$ (F658N) observations were made for 5000 seconds with WFPC2 over three orbits in November 2007. B, V, and I (F475W, F555W, F814W) observations were made with WFC3 in November 2010 with 1010, 696, and 800 second exposure times, respectively. These images were processed and combined using standard PyRAF and IRAF procedures; the combined image can be seen in Figure 1. The faintest star that can be seen is at V=26.9, at the $5 \sigma$ detection level.

Any leftover ex-companion star must still be located near the center of the remnant, so an accurate determination of the center is the first step in finding the ex-companion. We used three independent data sets to measure the center of the remnant: the $\mathrm{H} \alpha$ remnant edges, the X-ray remnant edges, and the distribution of the central light of the $\mathrm{H} \alpha$ shell. For the $\mathrm{H} \alpha$ edges, we constructed a series of nine sets of perpendicular bisectors, each of which provided a measurement of the center. These nine points were averaged to find the new center measurement, and the procedure was iterated to get a final value. We take the RMS scatter of the nine points as the $1 \sigma$ error bars. The edge points generated by this procedure can be used to define a long and short axis of the remnant: the radius in the direction of the long axis is $16.0^{\prime \prime}$ (oriented $18^{\circ} \pm 3^{\circ}$ west of north) and the radius in the direction short axis is $14.6^{\prime \prime}$. The center found using this method is RA=05:09:31.144, $\mathrm{DEC}=-67: 31: 17.17(\mathrm{~J} 2000)$, with $\sigma_{\text {short }}=0.18^{\prime \prime}$ and $\sigma_{\text {long }}=0.37^{\prime \prime}(\mathrm{J} 2000)$.

The same procedure was used to obtain the center from the X-ray shell edges, using Xray images found in Warren \& Hughes (2004). There are three separate images, for three different emission lines $(\mathrm{O}, \mathrm{Fe} \mathrm{L}$, and $\mathrm{Si}$ ), but since they are at a lower resolution than the $\mathrm{H} \alpha$ image, we only used three sets of bisectors per image, for a total of nine measured $\mathrm{X}$-ray centers. Again they were averaged, and the RMS scatter is reported as the $1 \sigma$ error on the measurement. The X-ray center is $\mathrm{RA}=05: 09: 31.195, \mathrm{DEC}=-67: 31: 17.11$ (J2000), with $\sigma_{\text {short }}=0.26 "$ and $\sigma_{\text {long }}=0.26 "$ ". 
The third method used the faint $\mathrm{H} \alpha$ light in the central region of the remnant. The $\mathrm{H} \alpha$ flux is faintest near the center, falling off from its peak at the edges, where the filaments are easily visible. We measure the brightness of $20 \times 20$ pixel regions tiled across the center of the remnant and fit them to a flux model. A similar set of tiles measured outside the remnant provides the uncertainty (from the RMS scatter) as well as the background flux. Using a chi-square fit, we find the center to be at $\mathrm{RA}=05: 09: 31.342, \mathrm{DEC}=-67: 31: 18.34$ $(\mathrm{J} 2000)$, with $\sigma_{\text {short }}=0.54^{\prime \prime}$ and $\sigma_{\text {long }}=0.60^{\prime \prime}$.

We take the weighted average of these three centers as our final geometric center, which gives $\mathrm{RA}=05: 09: 31.208, \mathrm{DEC}=-67: 31: 17.48$ (J2000) with $1 \sigma$ uncertainties of $0.14^{\prime \prime}$ in the direction of the short axis and $0.20^{\prime \prime}$ in the direction of the long axis.

\section{Offsets From the Geometric Center}

The position of any ex-companion star might be slightly offset from the geometric center of the remnant due to a number of factors. The star will have a significant amount of proper motion due to the orbital velocity it had as a member of the binary system, as well as kicks from the SN explosion itself. The kicks will be relatively small (Marietta et al. 2000; Canal et al. 2001; Pan et al. 2010) and the orbital velocity depends primarily on the stellar radius (for Roche lobe-filling companions), so we calculate separate offsets for red giant, subgiant, and main sequence stars. We also account for the motion of the instantaneously-ejected shell. The distribution of the distances from the geometric center is non-Gaussian, so we report the $99.73 \%$ (i.e. $3 \sigma$ ) containment probabilities. The expected proper motion of main sequence, subgiant, and red giant companions is 650 , 430 , and $170 \mathrm{~km} \mathrm{~s}^{-1}$. At the distance of the LMC, the highest velocity case corresponds to a proper motion of $0.0027^{\prime \prime}$ per year. For our 400 year old remnant, this gives a total maximal offset of $1.1^{\prime \prime}$.

We also must consider asymmetries in the shell expansion caused by non-sphericallysymmetric WD burning and/or enhanced/depleted circumstellar material around the original binary system. The observed ellipticity of the shell can be used to measure both of these effects. SNR 0509-67.5 has an axial ratio of $0.913 \pm 0.009$, and we can clearly determine the cause of this ellipticity by looking at the Spitzer $24 \mu$ images published by Borkowski et al. (2006), which show an excess of swept-up material in the west-southwest quadrant. The offset from geometric center is $1.39^{\prime \prime} \pm 0.14^{\prime \prime}$ and therefore the explosion site is at $\mathrm{RA}=05: 09: 30.976, \mathrm{DEC}=-67: 31: 17.90$, with $1 \sigma$ uncertainties of $0.21^{\prime \prime}$ and $0.20^{\prime \prime}$ in the directions of the long and short axes, respectively.

\section{Implications}

The central error ellipse (representing $3 \sigma$ containment) of SNR 0509-67.5 is empty of all point sources to the limiting magnitude of $\mathrm{V}=26.9$. The nearest red giant, subgiant, and possible MS companion are $7.4^{\prime \prime}, 5.8^{\prime \prime}$, and $2.9^{\prime \prime}$ from the center, respectively. There is a faint nebulous region visible within the error circle, but the excellent angular resolution of HST prevents the possibility of this nebula hiding a point source within the remnant. This nebula is likely an irregular background galaxy, but the coincidence with the explosion site is suggestive that it might be related to the supernova, perhaps low-velocity ejecta or the remains of a disrupted secondary WD (Piersanti et al. 2003).

The lack of a red giant within the error ellipse precludes the possibility of a symbiotic progenitor. The lack of either a red giant or a subgiant precludes the possibility of a recurrent nova progenitor. The lack of either a subgiant or any main sequence star brighter than $\mathrm{V}=22.7$ precludes the possibility of a persistent supersoft X-ray source progenitor or 


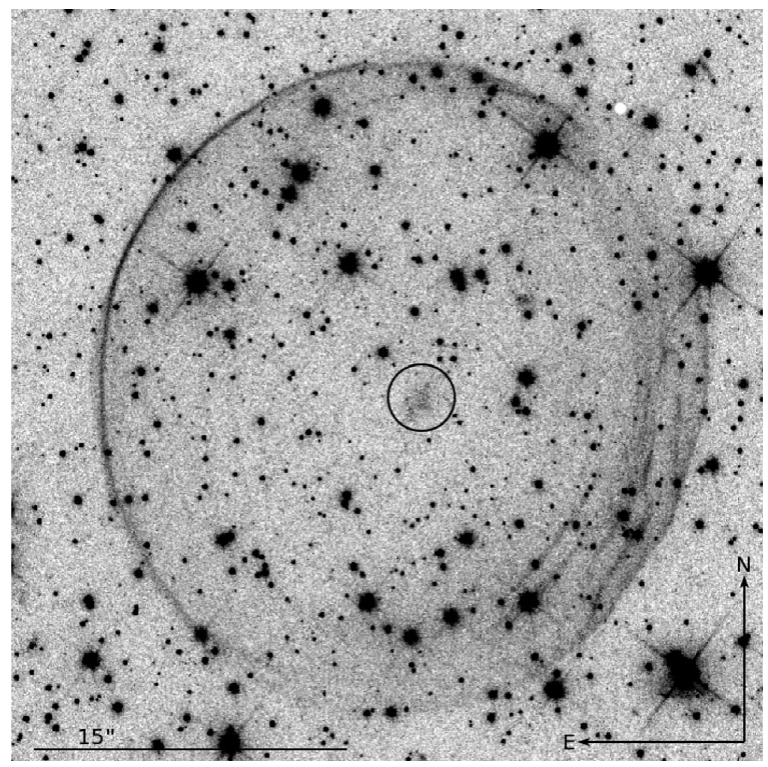

Figure 1. This combined $\mathrm{H} \alpha+$ BVI HST image shows the full SNR 0509-67.5 remnant and the $99.73 \%$ containment ellipse. Any possible ex-companion should be located within this ellipse, but it contains no point sources down to the $5 \sigma$ limiting magnitude of $\mathrm{V}=26.9$. We can exclude all published SD models and are left with the conclusion that SNR 0509-67.5 must have had a DD progenitor.

any other published SD model. We conclude that the only remaining possible progenitor model is that of a double degenerate (double white dwarf) system. We note that this result is not intended to be extrapolated to all known SNe Ia, but that the evidence for this particular system is very strong indeed.

\section{References}

Borkowski, K. J., et al. 2006, ApJ, 642, L141

Canal, R., Mendez, J., \& Ruiz-Lapuente, P. 2001, ApJ, 550, L53

González Hernández, J. I., Ruiz-Lapuente, P., Filippenko, A. V., Foley, R. J., Gal-Yam, A., \& Simon, J. D. 2009, ApJ, 691, 1

Hamuy, M., Phillips, M. M., Suntzeff, N. B., Schommer, R. A., Maza, J., \& Avilés, R. 1996, AJ, 112, 2391

Hughes, J. P., et al. 1995, ApJ, 444, L81

Ihara, Y., Ozaki, J., Doi, M., Shigeyama, T., Kashikawa, N., Komiyama, K., \& Hattori, T. 2007, $P A S J, 59,811$

Kerzendorf, W. E., Schmidt, B. P., Asplund, M., Nomoto, K., Podsiadlowski, P., Frebel, A., Fesen, R. A., \& Yong, D. 2009, ApJ, 701, 1665

Marietta, E., Burrows, A., \& Fryxell, B. 2000, ApJSupp, 128, 615

Pan, K.-C., Ricker, P. M., \& Taam, R. E. 2010, ApJ, 715, 78

Perlmutter, S., et al. 1999, ApJ, 517, 565

Piersanti, L., Gagliardi, S., Iben, Jr., I., \& Tornambé, A. 2003, ApJ, 583, 885

Rest, A., et al. 2005, Nature, 438, 1132

Rest, A., et al. 2008, ApJ, 680, 1137

Riess, A. et al. 1998, AJ, 116, 1009

Ruiz-Lapuente, P., et al. 2004, Nature, 431, 1069

Warren, J. S. \& Hughes, J. P. 2004, ApJ, 608, 261

Zaritsky, D., Harris, J., Thompson, I. B., \& Grebel, E. K. 2004, AJ, 128, 1606 